$63^{\text {ème }}$ Congrès de la SFCO, 03010 (2015)

DOI:10.1051/sfco/20156303010

(C) Owned by the authors, published by EDP Sciences, 2015

\title{
Nævus mélanocytaire composé palatin chez un enfant de 3 ans
}

\author{
Cloitre $\mathrm{A}^{*, * *}$, Boralévi $\mathrm{F}^{* * *}$, Catros $\mathrm{S}^{*}$, Fricain $\mathrm{JC}^{*}$ \\ * Service d'Odontologie, CHU Pellegrin, Bordeaux \\ ** Service d'Odontologie, CHU Hôtel-Dieu, Nantes \\ *** Service de Dermatologie, CHU Pellegrin, Bordeaux
}

Mots clés : muqueuse orale, nævus mélanocytaire, lésion pigmentée, enfant.

Introduction : Les nævi mélanocytaires oraux (NMO) sont des tumeurs mélanocytaires bénignes rares de la muqueuse orale qui peuvent être congénitales ou acquises. Leur incidence est évaluée à 4 cas pour 10 millions d'habitants par an et 500 cas ont été rapportés dans la littérature anglophone (Meleti 2007, 2008). Leur étiologie et leur pathogenèse restent largement méconnues. Les NMO sont plus fréquemment retrouvés chez les femmes $(\mathrm{H} / \mathrm{F}=0,6)$ entre la $3^{\text {ème }}$ et la $4^{\text {ème }}$ décennie (Meleti 2007, Buchner 1990 \& 2004). Les NMO ont une localisation préférentielle au palais comme les mélanomes de la muqueuse buccale (MMB) et peuvent revêtir un aspect clinique similaire.

Observation : Un enfant de 3 ans a consulté un pédiatre suite à la découverte fortuite d'une pigmentation palatine par ses parents. La lésion était asymptomatique. Les antécédents étaient une naissance à 37 semaines d'aménorrhée, une bronchiolite, une varicelle. Un traitement antifongique avait été prescrit pendant 15 jours, sans amélioration puis un dermatologue a ensuite adressé le patient au CHU. L'examen clinique a retrouvé une lésion pigmentée inhomogène brune de 2,5 cm de diamètre, aux bords irréguliers, plane et lisse, située au niveau de la muqueuse palatine gauche. Les aires ganglionnaires cervico-faciales étaient libres. Une biopsie a été réalisée sous anesthésie générale compte-tenu du jeune âge du patient. L'analyse histologique (HES, IHC protéine S-100) a mis en évidence une prolifération mélanocytaire regroupée en thèques au sein du chorion et à un moindre degré dans l'épithélium, évoquant un NMO composé. L'exérèse de la lésion n’a pas été nécessaire. Une surveillance clinique annuelle a été instaurée.

Discussion : Les diagnostics différentiels des NMO incluent les autres lésions pigmentées solitaires de la muqueuse orale qu'elles soient mélanocytaires (pigmentations ethniques, macules mélanocytaires, mélanoacanthomes, mélanomes, proliférations mélanocytaires atypiques) ou non mélanocytaires (pigments métalliques ou hématogènes). Le MMB, bien que rare, est une tumeur mélanocytaire maligne agressive, au pronostic sombre, dont la suspicion justifie une biopsie systématique. La liste ABCDE (Asymétrie, Bords irréguliers, Couleur inhomogène, Diamètre $>6 \mathrm{~mm}$, Evolution) a été proposée secondairement pour le dépistage des MMB (Meleti 2007 \& 2008). Dans le cas décrit, la lésion siégeait au palais et les critères ABCD étaient présents faisant suspecter un MMB mais le jeune âge du patient était peu compatible. Le diagnostic est histologique. Les NMO ont été suspectés d'être des précurseurs des MMB à cause de l'existence dans $30 \%$ des MMB d'une lésion pigmentée préexistante de nature indéterminée (Meleti 2007). A ce jour, le potentiel de transformation maligne des NMO n'a pas été déterminé et aucun cas de NMO dysplasique n’a été rapporté. Les NMO étant bénins, l'exérèse est seulement nécessaire en cas de gêne, néanmoins elle est généralement pratiquée car ces lésions sont rares, souvent de taille réduite, indiscernables cliniquement d'un mélanome débutant et non récidivantes.

This is an Open Access article distributed under the terms of the Creative Commons Attribution License 4.0, which permits unrestricted use, distribution, and reproduction in any medium, provided the original work is properly cited. 
$63^{\text {ème }}$ Congrès de la SFCO

Nom et adresse du conférencier

Alexandra CLOITRE

Service d'Odontologie, CHU Pellegrin

Bordeaux (France)

alexandra.cloitre@hotmail.fr 